\title{
Automated Multiple View Inspection Based on Uncalibrated Image Sequences
}

\author{
Domingo Mery and Miguel Carrasco \\ Departamento de Ciencia de la Computación \\ Pontificia Universidad Católica de Chile \\ Av. Vicuña Mackenna 4860(143), Santiago de Chile \\ dmery@ing.puc.cl
}

\begin{abstract}
The Automated Multiple View Inspection (AMVI) has been recently developed for automated defect detection of manufactured objects. The approach detects defects by analysing image sequences in two steps. In the first step, potential defects are automatically identified in each image of the sequence. In the second step, the potential defects are tracked in the sequence. The key idea of this strategy is that only the existing defects (and not the false detections) can be successfully tracked in the image sequence because they are located in positions dictated by the motion of the test object. The AMVI strategy was successfully implemented for calibrated image sequences. However, it is not simple to implement it in industrial environments because the calibration process is a difficult task and unstable. In order to avoid the mentioned disadvantages, in this paper we propose a new AMVI strategy based on the tracking of potential detects in uncalibrated image sequences. Our approach tracks the potential defects based on a motion model estimated from the image sequence self. Thus, we obtain a motion model by matching structure points of the images. We show in our experimental results on aluminium die castings that the detection is promising in uncalibrated images by detecting $92.3 \%$ of all existing defects with only 0.33 false alarms per image.
\end{abstract}

Keywords: defect detection, automated visual inspection, multiple view geometry.

\section{Introduction}

Recently, a new methodology, called the Automated Multiple View Inspection (AMVI), has been developed for automated defect detection 11. In contrast to the classic inspection methods that analyse individual images, AMVI detects defects by analysing image sequences. Thus, AMVI is similar to the way a (human) inspector examines a test object: first, the inspector detects anomalous details in an image sequence obtained from the test object in motion; and second, the inspector tracks in the image sequence the irregularities detected in the first step. If the inspector can track them, i.e., if the irregularities are visible 
among the image sequence, he or she classifies the test object as defectively. Similarly, the suggested computer-aided method AMVI is able to detect defects in two steps. In the first step, called identification, potential defects are automatically identified in each image of the sequence using a single filter and no a priori knowledge of the structure of the test object. In the second step, called tracking, an attempt is made to track the identified potential defects in the image sequence. Therefore, only the existing defects (and not the false detections) can be successfully tracked in the image sequence because they are located in positions dictated by the motion of the test object. Thus, two or more views of the same object taken from different viewpoints can be used to confirm and improve the diagnostic done by analysing only one image. A similar idea is also used by radiologists that analyse two different view X-rays of the same breast to detect cancer in its early stages. Hence, the number of cancers flagged erroneously and missed cancers may be greatly reduced (see for example [2], where a novel method that finds automatically correspondences in two different views of the breast is presented).

The exploitation of the multiple view analysis represents a new methodology in the automated visual inspection. Indeed, this multiple view strategy is opening up new possibilities in this field by taking into account the useful information about the correspondence between the different views of the test object.

The AMVI strategy was implemented in 1 for automated inspection of aluminium die castings using calibrated radioscopic image sequences. In this case, the projection model 3D $\rightarrow 2 \mathrm{D}$ is estimated off-line using a calibration object, and the potential defects are tracked on-line according to the principles of multiple view geometry [3, where the geometric constraints between different views of the sequence can be easily established using multi-focal tensors. The preliminary results obtained using AMVI methodology are promising in calibrated image sequences achieving impressive discrimination of false alarms while detecting the real defects of the object test. However, it is not simple to implement it in industrial environments because the calibration process is a difficult task and the vibrations of the imaging system induce inaccuracies in the estimated parameters of the multiple view geometric model. Thus, the calibration is not stable and the imaging system must be calibrated periodically.

In order to avoid the mentioned disadvantages, in this paper we propose a new AMVI strategy based on the tracking of potential detects in uncalibrated image sequences. Hence, it is not necessary to calibrate the imaging system. The reason why uncalibrated images can be used is because there are several constraints characteristic of the inspection problem that simplify the tracking task. Our approach tracks the potential defects based on a motion model estimated from the image sequence self. By means of this motion model, the potential defects can be successfully tracked in the uncalibrated image sequence.

In this paper we present the mentioned new methodology based on uncalibrated image sequences. The rest of the paper is organised as follows: Section 2 explains our approach for uncalibrated AMVI. Section 3 shows preliminary 
results obtained with the proposed methodology. Finally, Section 4 gives concluding remarks and perspectives for future works.

\section{Uncalibrated AMVI}

The main objective of our research is to perform a robust automated visual inspection based on the tracking of potential detects without calibrating the imaging system. The reason why uncalibrated images can be used is because there are several constraints characteristic of our inspection problem that can be considered to perform the tracking in an uncalibrated way: e.g., the scene captured by an image of the sequence consists of only one rigid object in motion, there is no significant frame to frame motion and the $2 \mathrm{D}$ trajectories are smooth, the velocity of the test object is constant, and generally the motion of the test object is only rotational or translational. Since the aim of AMVI is to track the potential defects only (and not to estimate the structure of the test object), we propose to track the potential defects without computing the $3 \mathrm{D} \rightarrow 2 \mathrm{D}$ model.

Our approach estimates a motion model using a simple methodology: i) it identifies structure points in each image of the sequence; ii) it finds corresponding structure points between consecutive frames of the sequence; and iii) it estimates the fundamental matrices from the corresponding points using a robust algorithm. By means of this motion model, the potential defects can be successfully tracked in the uncalibrated image sequence. Thus, the key idea of the uncalibrated AMVI is that the information obtained from the tracking of structure points can be used to track the potential defects in the sequence.

The proposed method for uncalibrated AMVI can be structured in two general steps (see Fig. 10): A) motion estimation and B) defects detection. The second step is similar to the calibrated AMVI method explained in previous section. In the following, both steps are described in further detail.

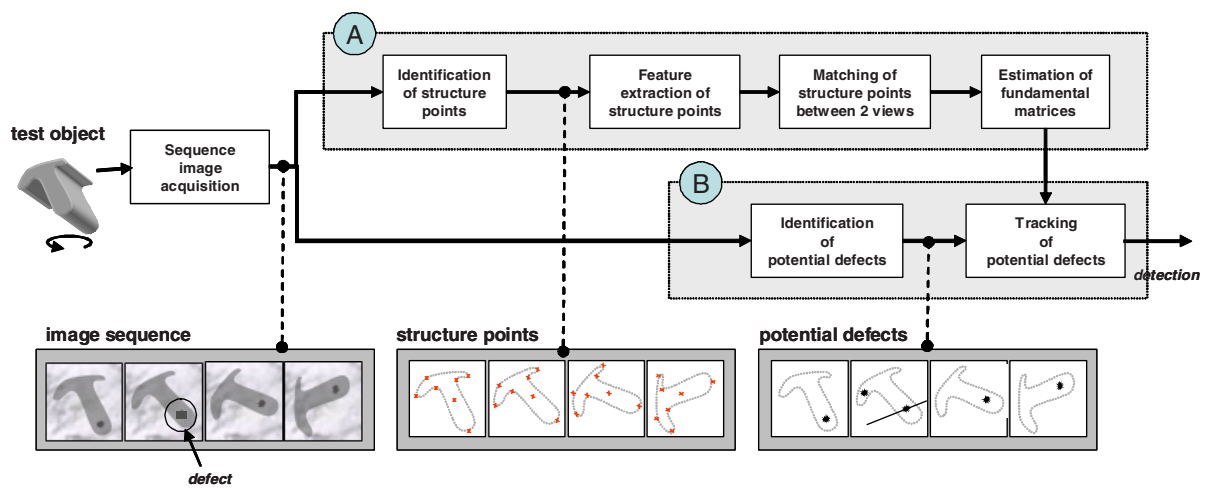

Fig. 1. Block diagram of the uncalibrated automated multiple view inspection: A) estimation of motion model, B) detection of defects 
A.1) Identification of structure points: The whole test object is segmented using the Otsu's approach [4], where the foreground is separated from the background using a global threshold. In this case, it is assumed that the intensity of the object is clearly differentiated from the background, i.e., the image presents a bimodal histogram. The segmented regions of the object are labelled. For each labelled region the centre of mass and the external points (top-left, top-right, right-top, right-bottom, bottom-right, bottom-left, left-bottom and left-top) are defined as structure points of the object. Incomplete regions are not considered.

A.2) Feature extraction of structure points: In order to match the identified structure points, certain features must be measured. In our approach, the coordinates, and the mean grey level of the neighbourhood are extracted for each identified structure point.

A.3) Matching of structure points: Since the motion of the test object is slow, i.e., the trajectories in the sequence are smooth, the structure points in two consecutive frames can be easily matched by considering the nearness of the structure points and the similarity in the grey level.

A.4) Estimation of fundamental matrices: Structure points can be erroneously matched in previous step. In addition, for rotated circular regions the external points in different views are not necessarily corresponding points. For this reason, a robust algorithm that estimates the fundamental matrix is required. We use the RANSAC approach [3] that estimates the fundamental matrix without considering outliers (mismatch画gs).

B.1) Identification of potential defects: The segmentation of potential defects identifies regions in each image of the sequence that may correspond to real defects. Two general characteristics of the defects are used to identify them: a) a defect can be considered as a connected subset of the image, and b) the grey level difference between a defect and its neighbourhood is significant. The potential defects are identified without a-priori knowledge. First, a Laplacian-of-Gaussian (LoG) kernel and a zero crossing algorithm [5] are used to detect the edges of the X-ray images. The LoG-operator involves $\bigoplus$ Gaussian lowpass filter which is a good choice for the pre-smoothing of our noisy images that are obtained without frame averaging. The resulting binary edge image should produce at real defects closed and connected contours which demarcate regions. However, a defect may not be perfectly enclosed if it is located at an edge of a regular structure as shown in Fig. 2c. In order to complete the remaining edges of these defects, a thickening of the edges of the regular structure is performed as follows: a) the gradient of the original image is calculated (see Fig. 2d); b) by thresholding the gradient image at a high grey level a new binary image is obtained; and c) the resulting image is added to the zero crossing image (see Fig. 2e). Afterwards, each closed region is segmented. In order to identify the potentiकidefects, features are 

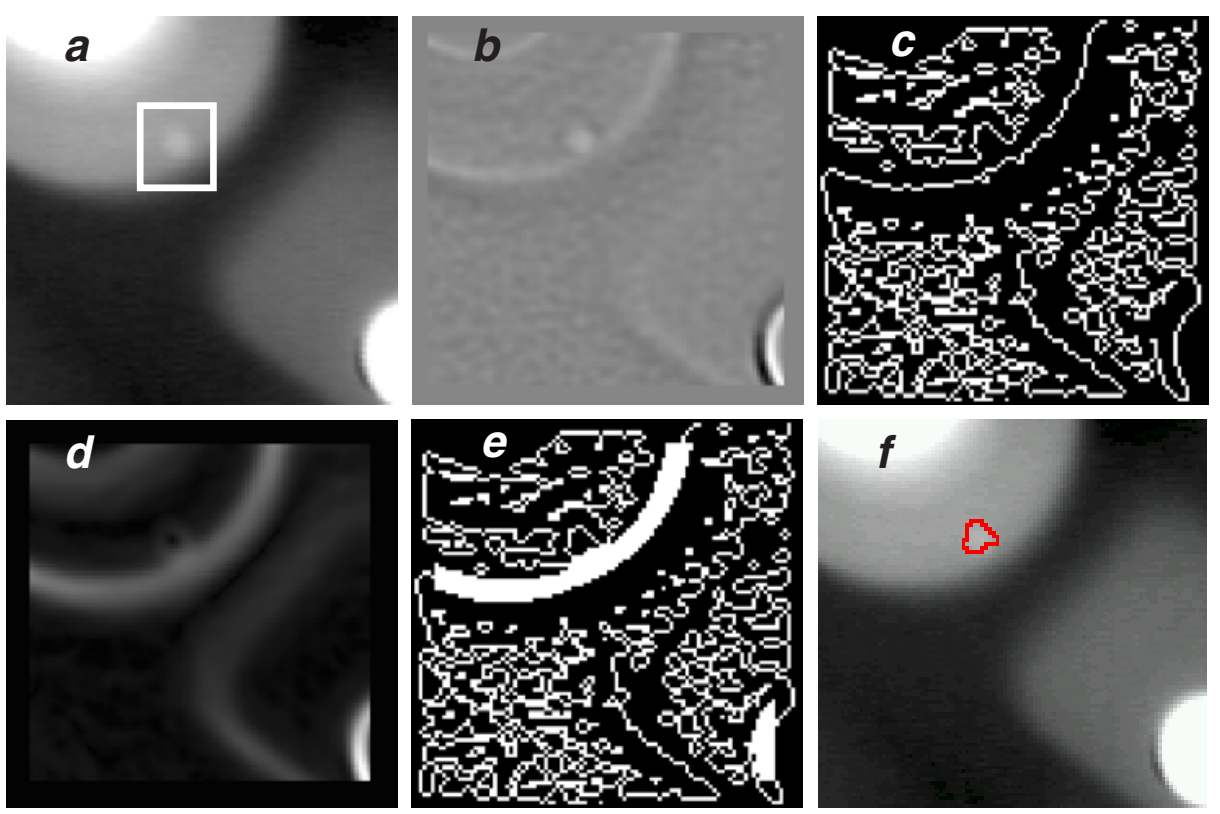

Fig. 2. Detection of flaws: a) radioscopic image with a small flaw at an edge of a regular structure, b) Laplacian-filtered image with $\sigma=1.25$ pixels (kernel size $=11 \times 11$ ), c) zero crossing image, d) gradient image, e) edge detection after adding high gradient pixels, and f) detected flaw using the variance of the crossing line profile

extracted from crossing line profiles of each segmented region [6]. Crossing line profiles are grey level profiles along straight lines crossing each segmented region in the middle. If the variance of the crossing line profiles is high, the segmented region is classified as potential defect. This is a very simple detector of potential defects with a large number of false alarms flagged erroneously. However, the advantages are as follows: a) it is a single detector (it is the same detector for each image), b) it is able to identify potential defects independent of the placement and the structure of the test object, i.e., without a-priori information of the design structure of the test object, and c) the detection rate of real defects is very high (more than $90 \%$ ).

B.2) Tracking of potential defects: Tracking requires the position of the centre of mass of each detected potential defect. In this work, $\mathrm{a}=(a, p)$ will denote the identified potential defect $a$ in image $p$. It is assumed that the image sequence has $N$ images $(1 \leq p \leq N)$ and $n_{p}$ points were identified in image $p\left(1 \leq a \leq n_{p}\right)$. The position of potential defect $\mathrm{a}=(a, p)$ is arranged in a position vector $\mathbf{m}_{p}^{a}$. One obtains then the position vector $\mathbf{m}_{p}^{a}=\left(x_{p}^{a}, y_{p}^{a}\right)$. This step matches two potential defects (of two views), potential defect $\mathrm{a}=(a, p)$ with potential defect $\mathrm{b}=(b, q)$, for $p \neq q$, if $\mathbf{m}_{p}^{a}$ and $\mathbf{m}_{q}^{b}$ satisfy the epipolar constraint. In addition, a similarity condition is used, i.e., the matching is established if the potential defects are similar enough. To evaluate this criterion a degree of 
similarity is calculated as the Euclidean distance between the normalised feature vectors of the potential defects. In this case, the features area and grey level value of the potential defects are used to establish the similarity. If the false alarms cannot be eliminated with the tracking in two views, a tracking in three and four views using trifocal tensors should be used (see for example 77). Trifocal tensors can be estimated from the fundamental matrices 3 .

\section{$3 \quad$ Experimental Results}

In this section, we present results obtained from several radioscopic images of aluminium die castings using the proposed approach. For example, two different radioscopic images of an aluminium wheel, used in our experiments, are shown in Fig. 3. These images will be used to illustrate the steps of our method outlined in Section 2. The algorithm starts with the identification of structure points in each view (see Fig. 4). Afterwards, the position of each point and its mean grey level of a $3 \times 3$ centred mask are measured. These values are used to match the structure points in both views. Using the matched points, the fundamental matrix of these two views is estimated with a RANSAC algorithm. Fourteen obtained epipolar lines are shown in Fig. 3. We observe that the epipolar lines lie on the marked points successfully.

After the estimation of the fundamental matrix, we identify the potential defects in each image. The results are shown in Fig. 5 , We observe that left image has six potential defects (only two of them are real defects). On the other hand, right image has seven potential defects (only two of them are real defects). In both images, the existing defects are identified. However, there are nine false alarms (four in left image and five in right image) that do not correspond to real defects. The false alarms should be eliminated by the tracking step.
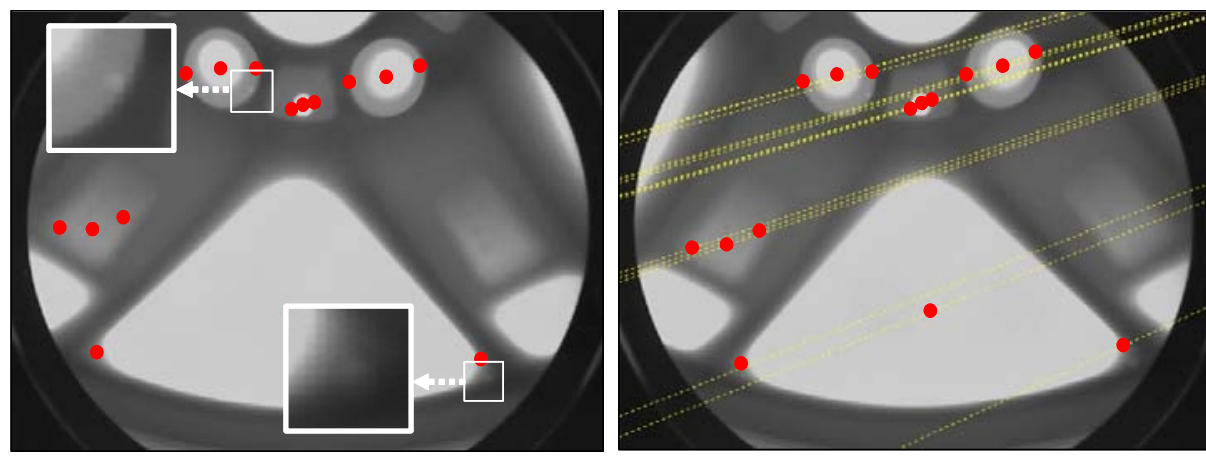

Fig. 3. Radioscopic images of a wheel using in our experiments. In each view, there are two defects (see white squares in left view). In addition, the obtained epipolar lines are drawn for fourteen points 

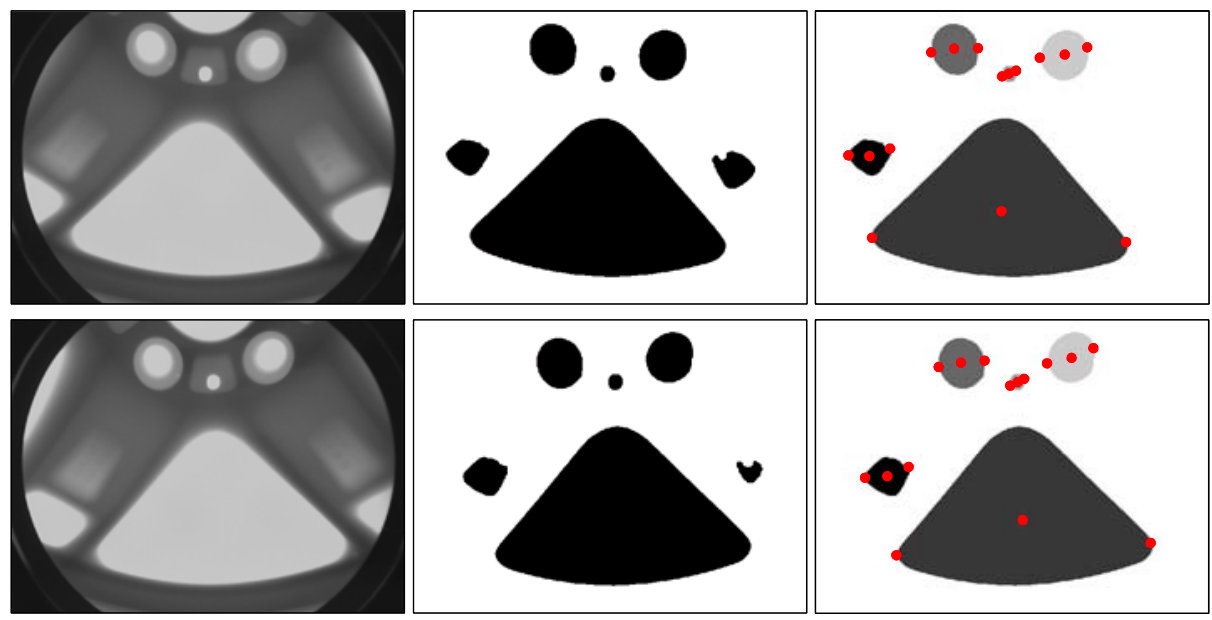

Fig. 4. Finding structure points in two views: left) original images, middle) segmentation after Otsu's method, right) extracted structure points
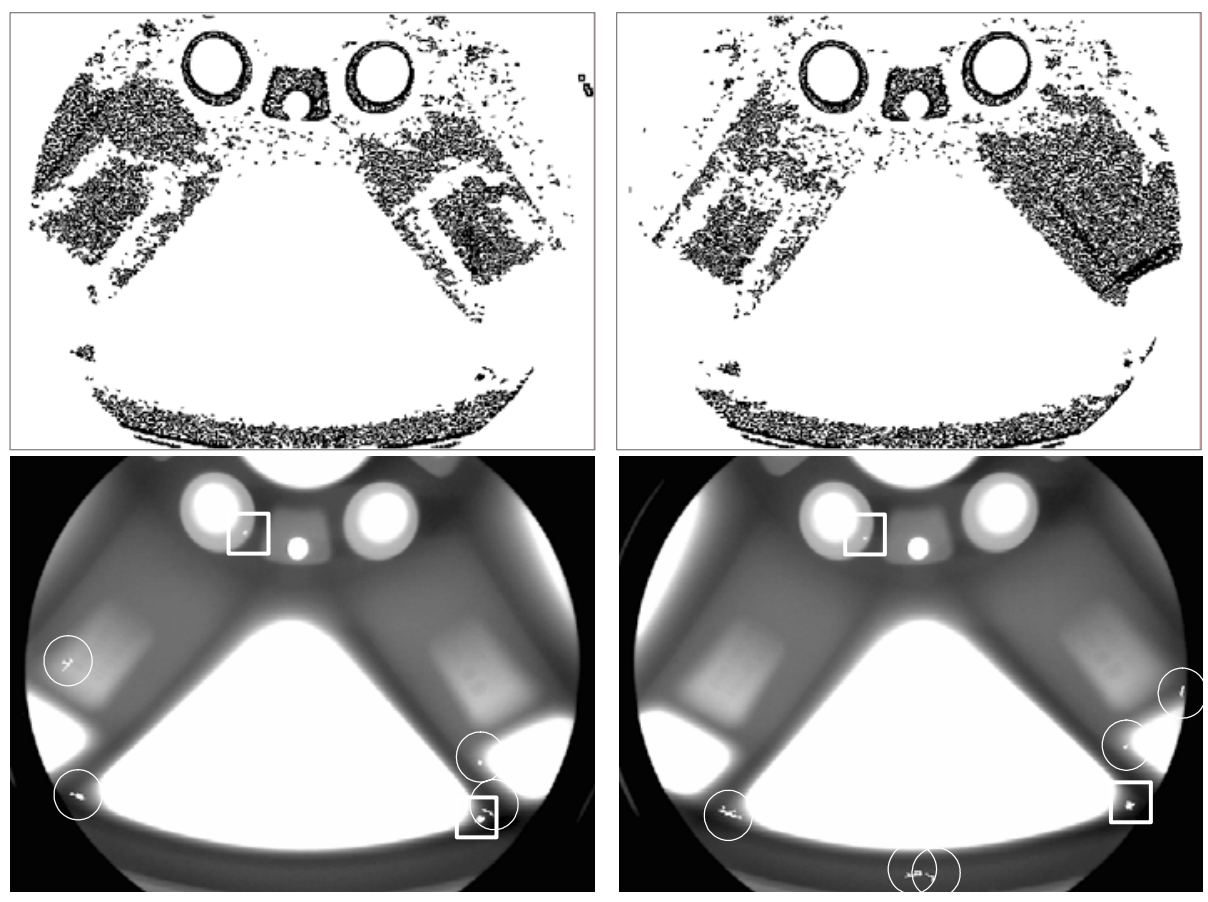

Fig. 5. Identification of potential defects in both views: top) segmented regions, bottom) potential defects after crossing line profile approach (squares: real defects, circles: false alarms) 
The results of the tracking step are shown in 6 In this step, we try to find in right image the corresponding potential defects of the six potential defects of left image. In the AMVI strategy, the search for the corresponding potential defect in both images is restricted to the epipolar lines and to similar potential defects. The similarity of the candidates is evaluated by comparing their areas and mean grey values. In this approach, we eliminate those potential defects that cannot be matched. In this example, the existing defects are detected flagging only one false alarm.

In order to verify the performance of our method, a broader set of images were analised. In our experiments, radioscopic image sequences (without frame
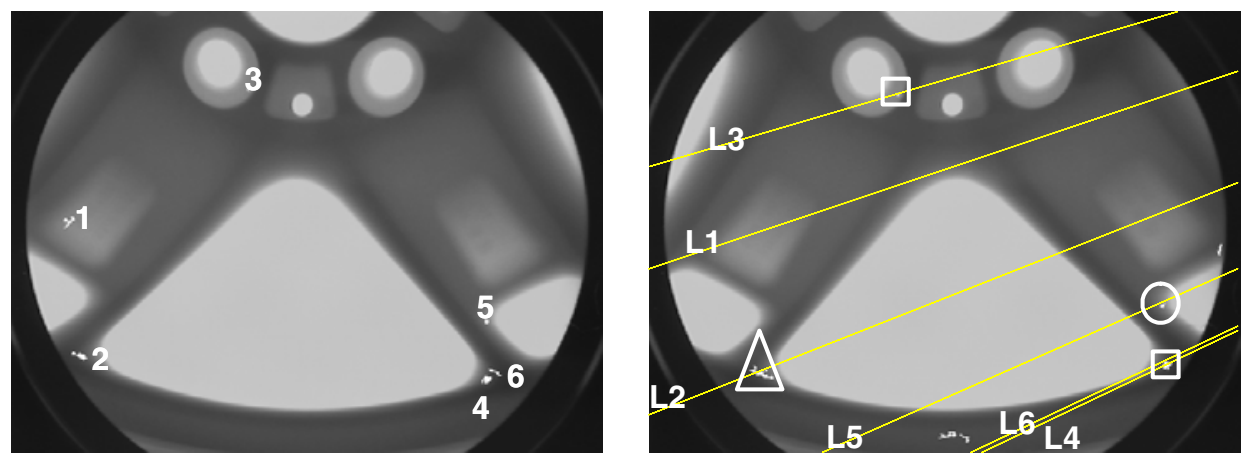

Fig. 6. Matching of potential defects in both images. The six epipolar lines of the potential defects of left image are denoted as L1, ... L6 in right image. Potential defects 1 and 6 cannot be matched (there is no potential defect on the epipolar lines), i.e., potential defects 1 and 6 are eliminated. The epipolar line L2 meets a potential defect in right image (see triangle), however both potential defects are not similar enough, i.e., potential defect 2 is eliminated. Potential defects 3, 4 and 5 fulfil epipolar and similarity conditions, i.e., they are detected as defects, however potential defect 5 is a false alarm. In this case, the existing defects are detected successfully (see squares) with one false alarm (see circle)
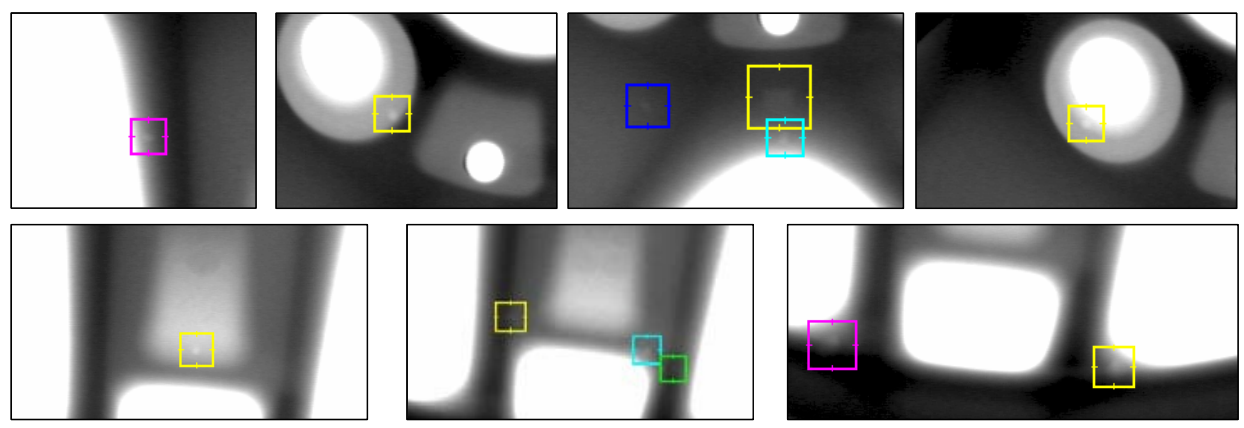

Fig. 7. Details of the radioscopic images used in our experiments. The squares in all images of this section were drawn intentionally to show the reader where the defects are located 
averaging) of aluminium wheels with twelve known flaws were inspected. Three of these defects were existing blow holes $(\emptyset=1.5 \sim 7.5 \mathrm{~mm})$. They were initially detected by a visual (human) inspection. The remaining nine flaws were produced by drilling small holes $(\emptyset=2.0 \sim 4.0 \mathrm{~mm})$ in positions of the casting which were known to be difficult to detect. Some details of the radioscopic images showing the defects are presented in Fig. 7

We test this approach in twelve stereo images, i.e., in 24 radioscopic images of $572 \times 768$ pixels. Since there are twelve defects viewed in different images of the sequences, the total number of the imaged flaws was 39 in the 24 images. In this experiment, the identification of potential defects was perfect, i.e., all existing defects were successfully segmented. However, there were many false alarms. The results of the identification are summarised in Table 1. On average, there were 3.25 defects and 3.46 false alarms per image, i.e., $51.5 \%$ of the identified potential defects were false alarms.

After the tracking step, $92.3 \%$ of the potential defects were successfully detected with only 0.33 false alarms per image. Table 2 compares the results obtained in the identification and tracking steps. We observe that the tracking step is able to filter out $90.0 \%$ (from 41.5 to 4 ) of the false alarms eliminating only $7.7 \%$ (from 39 to 36 ) of the existing defects.

Table 1. Performance of the identification of potential defects

\begin{tabular}{|l|c|c|c|}
\hline Images & Number of images & Detected defects/image & False alarms/image \\
\hline Left images & 12 & $39 / 12=3.25$ & $40 / 12=3.33$ \\
Right images & 12 & $39 / 12=3.25$ & $43 / 12=3.58$ \\
All images & 24 & $78 / 24=3.25$ & $83 / 24=3.46$ \\
\hline
\end{tabular}

Table 2. Performance of the tracking of potential defects

\begin{tabular}{|l|c|c|c|c|c|c|}
\hline Step & $\begin{array}{c}\text { Detected } \\
\text { defects }\end{array}$ & $\begin{array}{c}\text { No detected } \\
\text { defects }\end{array}$ & $\begin{array}{c}\text { False } \\
\text { alarms }\end{array}$ & $\begin{array}{c}\text { Detection } \\
\text { performance }\end{array}$ & $\begin{array}{c}\text { False alarm } \\
\text { rate }\end{array}$ & $\begin{array}{c}\text { False alarms } \\
\text { per image }\end{array}$ \\
\hline Identification & 39 & 0 & 41.5 & $100 \%$ & $51.5 \%$ & 3.46 \\
Tracking & 36 & 3 & 4 & $92.3 \%$ & $10.0 \%$ & 0.33 \\
\hline
\end{tabular}

\section{Concluding Remarks}

In this paper we presented a new automated multiple view inspection strategy based on the tracking of potential detects in uncalibrated image sequences. Our approach tracks the potential defects based on a motion model estimated from the image sequence self. Since no calibration is required, we believe that the implementation of the automated multiple view inspection will be now possible in industrial environments.

We have shown in our experimental results on aluminium die castings that the detection is promising in uncalibrated images by detecting $92.3 \%$ of all existing defects with only 0.33 false alarms per image. However, since the performance 
of the method has been verified on only 24 images, an evaluation on a broader data base is necessary.

In our experiments, we use a tracking based on only two views because the quote of false alarms is low. Nevertheless, as future work the tracking step will be performed using three and four views in order to increase the performance of the algorithm.

\section{Acknowledgment}

This work was supported by FONDECYT - Chile under grant no. 1040210.

\section{References}

1. Mery, D., Filbert, D.: Automated flaw detection in aluminum castings based on the tracking of potential defects in a radioscopic image sequence. IEEE Trans. Robotics and Automation 18 (2002) 890-901

2. Kita, Y., Highnam, R., Brady, M.: Correspondence between different view breast X-rays using curved epipolar lines. Computer, Vision and Understanding 83 (2001) $38-56$

3. Hartley, R.I., Zisserman, A.: Multiple view geometry in computer vision. Cambridge University Press (2000)

4. Haralick, R., Shapiro, L.: Computer and robot vision. Addison-Wesley Publishing Co., New York (1992)

5. Castleman, K.: Digital image processing. Prentice-Hall, Englewood Cliffs, New Jersey (1996)

6. Mery, D.: Crossing line profile: a new approach to detecting defects in aluminium castings. Lecture Notes in Computer Science 2749 (2003) 725-732

7. Mery, D., Ochoa, F., Vidal, R.: Tracking of points in a calibrated and noisy image sequence. Lecture Notes in Computer Science 3211 (2004) 647-654 